\title{
СВОЙСТВА ИНТЕГРИРУЕМОСТИ ФУНКЦИЙ С ЗАДАННЫМ ПОВЕДЕНИЕМ ФУНКЦИЙ РАСПРЕДЕЛЕНИЯ И НЕКОТОРЫЕ ПРИЛОЖЕНИЯ ${ }^{1}$
}

\begin{abstract}
А. А. Ковалевский
Установлено, что если функция распределения измеримой функции $v$, заданной на ограниченной области $\Omega \subset \mathbb{R}^{n}(n \geqslant 2)$, при достаточно больших $k$ удовлетворяет оценке meas $\{|v|>k\} \leqslant k^{-\alpha} \varphi(k) / \psi(k)$, где $\alpha>0, \varphi:[1,+\infty) \rightarrow \mathbb{R}-$ неотрицательная невозрастающая измеримая функция такая, что интеграл функции $s \rightarrow \varphi(s) / s$ по $[1,+\infty)$ конечен, и $\psi:[0,+\infty) \rightarrow \mathbb{R}-$ положительная непрерывная функция с некоторыми дополнительными свойствами, то $|v|^{\alpha} \psi(|v|) \in L^{1}(\Omega)$. При этом функция $\psi$ может быть как ограниченной, так и неограниченной. Даны следствия соответствующих теорем для некоторых конкретных отношений функций $\varphi$ и $\psi$. В частности, рассмотрен случай, когда функция распределения измеримой функции $v$ при достаточно больших $k$ удовлетворяет оценке meas $\{|v|>k\} \leqslant C k^{-\alpha}(\ln k)^{-\beta}$, где $C, \alpha>0$ и $\beta \geqslant 0$. При этом усилен результат, полученный автором ранее для $\beta>1$, и в целом показано, как отличаются свойства интегрируемости функции $v$ в зависимости от того, какому из промежутков, $[0,1]$ или $(1,+\infty)$, принадлежит $\beta$. Рассмотрен также случай, когда функция распределения измеримой функции $v$ при достаточно больших $k$ удовлетворяет оценке $\operatorname{meas}\{|v|>k\} \leqslant C k^{-\alpha}(\ln \ln k)^{-\beta}$, где $C, \alpha>0$ и $\beta \geqslant 0$. Приведены примеры, показывающие точность полученных результатов в соответствующих шкалах классов, близких к $L^{\alpha}(\Omega)$. Наконец, даны приложения этих результатов к энтропийным и слабым решениям задачи Дирихле для нелинейных эллиптических уравнений второго порядка с правой частью из некоторых классов, близких к $L^{1}(\Omega)$ и определяемых с помощью логарифмической функции или ее двукратной композиции.
\end{abstract}

Ключевые слова: интегрируемость, функция распределения, нелинейные эллиптические уравнения, правая часть из классов, близких к $L^{1}$, задача Дирихле, слабое решение, энтропийное решение.

A. A. Kovalevsky. Integrability properties of functions with a given behavior of distribution functions and some applications.

We establish that if the distribution function of a measurable function $v$ given on a bounded domain $\Omega$ of $\mathbb{R}^{n}(n \geqslant 2)$ satisfies, for sufficiently large $k$, the estimate meas $\{|v|>k\} \leqslant k^{-\alpha} \varphi(k) / \psi(k)$, where $\alpha>0$, $\varphi:[1,+\infty) \rightarrow \mathbb{R}$ is a nonnegative nonincreasing measurable function such that the integral of the function $s \rightarrow \varphi(s) / s$ over $[1,+\infty)$ is finite, and $\psi:[0,+\infty) \rightarrow \mathbb{R}$ is a positive continuous function with some additional properties, then $|v|^{\alpha} \psi(|v|) \in L^{1}(\Omega)$. In so doing, the function $\psi$ can be bounded or unbounded. We give corollaries of the corresponding theorems for some specific ratios of the functions $\varphi$ and $\psi$. In particular, we consider the case where the distribution function of a measurable function $v$ satisfies, for sufficiently large $k$, the estimate meas $\{|v|>k\} \leqslant C k^{-\alpha}(\ln k)^{-\beta}$ with $C, \alpha>0$ and $\beta \geqslant 0$. In this case, we strengthen our previous result for $\beta>1$ and, on the whole, we show how the integrability properties of the function $v$ differ depending on which of the intervals $[0,1]$ or $(1,+\infty)$ contains $\beta$. We also consider the case where the distribution function of a measurable function $v$ satisfies, for sufficiently large $k$, the estimate meas $\{|v|>k\} \leqslant C k^{-\alpha}(\ln \ln k)^{-\beta}$ with $C, \alpha>0$ and $\beta \geqslant 0$. We give examples showing the accuracy of the obtained results in the corresponding scales of classes close to $L^{\alpha}(\Omega)$. Finally, we give applications of these results to entropy and weak solutions of the Dirichlet problem for nonlinear elliptic second-order equations with right-hand side in some classes close to $L^{1}(\Omega)$ and defined by the logarithmic function or its double composition.

Keywords: integrability, distribution function, nonlinear elliptic equations, right-hand side in classes close to $L^{1}$, Dirichlet problem, weak solution, entropy solution.

MSC: 26B35, 35J25, 35J60

DOI: $10.21538 / 0134-4889-2019-25-1-78-92$

\section{Введение}

По определению (см., например, $[1 ; 2])$ функция распределения измеримой функции $v$, заданной на ограниченной области $\Omega \subset \mathbb{R}^{n}(n \geqslant 2)$, - это соответствие $s \rightarrow \operatorname{meas}\{|v|>s\}, s \geqslant 0$.

\footnotetext{
${ }^{1}$ Работа выполнена при частичной поддержке Программы повышения конкурентоспособности ведущих университетов РФ (соглашение с Минобрнауки РФ 02.А03.21.0006 от 27 августа 2013 г.).
} 
По оценке значений функции распределения можно судить об интегрируемости на $\Omega$ исходной функции или некоторой зависящей от нее функции. Изучение этого вопроса представляет интерес, в частности, для выяснения свойств интегрируемости решений эллиптических уравнений и вариационных неравенств с правой частью из пространства $L^{1}(\Omega)$ или классов функций, близких к $L^{1}(\Omega)$. Так, например, в [2] для функции распределения энтропийного решения $u$ задачи Дирихле для нелинейного эллиптического уравнения второго порядка с правой частью $f \in L^{1}(\Omega)$ при $k>0$ получена оценка вида

$$
\operatorname{meas}\{|u|>k\} \leqslant C k^{-\alpha}
$$

с некоторыми положительными константами $C$ и $\alpha$, первая из которых зависит от $n$ (размерности пространства), показателя $p$, характеризующего рост коэффициентов уравнения, и нормы функции $f$ в $L^{1}(\Omega)$, а вторая зависит только от $n$ и $p$. Аналогичная оценка была установлена и для градиента энтропийного решения. Из полученных оценок следует интегрируемость определенных степеней модулей энтропийного решения и его градиента. В частности, из оценки $(0.1)$ вытекает, что $u \in L^{\lambda}(\Omega)$ для любого $\lambda \in(0, \alpha)$. Кроме того, в случае $p>2-1 / n$ установленные оценки обеспечивают принадлежность энтропийного решения пространствам Соболева с показателем, меньшим некоторого предельного.

Если функция распределения измеримой функции $v: \Omega \rightarrow \mathbb{R}$ при достаточно больших $k$ удовлетворяет оценке

$$
\operatorname{meas}\{|v|>k\} \leqslant C k^{-\alpha}(\ln k)^{-\beta},
$$

где $C, \alpha>0$ и $\beta>1$, или более общей оценке

$$
\operatorname{meas}\{|v|>k\} \leqslant k^{-\alpha} \varphi(k),
$$

где $\alpha>0$ и $\varphi:[1,+\infty) \rightarrow \mathbb{R}-$ неотрицательная невозрастающая измеримая функция такая, что интеграл функции $s \rightarrow \varphi(s) / s$ по $[1,+\infty)$ конечен, то, как показано, например, в [3;4], функция $v$ принадлежит $L^{\alpha}(\Omega)$. С использованием этих результатов в упомянутых работах были установлены условия на правую часть нелинейного эллиптического уравнения второго порядка, при которых энтропийное решение соответствующей задачи Дирихле и его градиент принадлежат некоторым предельным пространствам Лебега.

Опираясь на доказанное в [4] утверждение о принадлежности измеримой функции $v$ пространству $L^{\alpha}(\Omega)$, если для ее функции распределения верна оценка $(0.3)$ с указанной выше функцией $\varphi$, в настоящей работе изучим случай, когда функция распределения измеримой функции $v$ при достаточно больших $k$ удовлетворяет оценке

$$
\operatorname{meas}\{|v|>k\} \leqslant \frac{\varphi(k)}{k^{\alpha} \psi(k)},
$$

где $\alpha>0$ и $\varphi$ - такая же функция, как и выше, а $\psi$ - положительная непрерывная функция на $[0,+\infty)$ с некоторыми дополнительными свойствами. Оказывается, что в этом случае справедливо включение $|v|^{\alpha} \psi(|v|) \in L^{1}(\Omega)$. При этом функция $\psi$ может быть как ограниченной, так и неограниченной. Соответствующие результаты доказаны в разд. 1 (см. теоремы 1 и 2). Следствия этих общих результатов для некоторых конкретных отношений функций $\varphi$ и $\psi$ в оценке (0.4) приведены в разд. 2. В частности, рассмотрен случай, когда функция распределения измеримой функции $v$ удовлетворяет оценке $(0.2)$ с $C, \alpha>0$ и $\beta \geqslant 0$. При этом усилен результат, полученный в [3] для $\beta>1$, и в целом показано, как отличаются свойства интегрируемости функции $v$ в зависимости от того, какому из промежутков, $[0,1]$ или $(1,+\infty)$, принадлежит $\beta$ (см. следствия 1 и 2). Рассмотрен также случай, когда функция распределения измеримой функции $v$ удовлетворяет оценке $\operatorname{meas}\{|v|>k\} \leqslant C k^{-\alpha}(\ln \ln k)^{-\beta}$ с $C, \alpha>0$ и $\beta \geqslant 0$ (см. следствия 3 и 4). В разд. 3 приведены примеры, показывающие точность результатов предыдущего раздела в соответствующих шкалах классов, близких к $L^{\alpha}(\Omega)$. При этом под близостью класса функций $K$ к пространству $L^{\alpha}(\Omega)$ понимается, что выполняется одно 
из следующих условий: (i) $K \subset L^{\alpha}(\Omega)$ и $K \not \subset L^{\alpha+\varepsilon}(\Omega)$ для любого $\varepsilon>0$; (ii) $K \not \subset L^{\alpha}(\Omega)$ и $K \subset L^{\alpha-\varepsilon}(\Omega)$ для любого $\varepsilon \in(0, \alpha)$. Наконец, в разд. 4 даны приложения результатов разд. $2 \mathrm{~K}$ энтропийным и слабым решениям задачи Дирихле для нелинейных эллиптических уравнений второго порядка с правой частью из некоторых классов, близких к $L^{1}(\Omega)$ и определяемых с помощью логарифмической функции или ее двукратной композиции. В итоге усилены известные и получены новые результаты о свойствах интегрируемости модулей указанных решений.

\section{1. Общие теоремы}

Пусть $n \in \mathbb{N}, n \geqslant 2$, и пусть $\Omega$ - ограниченное открытое множество в $\mathbb{R}^{n}$.

Приведем предложение, на которое опираются последующие теоремы.

Предложение 1. Пусть $v: \Omega \rightarrow \mathbb{R}$ - измеримая функиия, и пусть $\varphi:[1,+\infty) \rightarrow \mathbb{R}-$ неотрицательная невозрастающая измеримая функиия. Пусть $\alpha>0 u k_{0} \geqslant 1$. Предположсим, что выполняются следующие условия:

(a) $\int_{1}^{+\infty} \frac{\varphi(s)}{s} d s<+\infty$;

(b) $\forall k \geqslant k_{0} \quad$ meas $\{|v|>k\} \leqslant k^{-\alpha} \varphi(k)$.

Тогда $v \in L^{\alpha}(\Omega)$.

Сформулированное предложение, по существу, совпадает с леммой 2.1 из [4] и в отдельном доказательстве не нуждается.

Перейдем к формулировке и доказательству теорем.

Теорема 1. Пусть $v: \Omega \rightarrow \mathbb{R}$ - измеримая функиия, $\varphi:[1,+\infty) \rightarrow \mathbb{R}-$ неотрицательная невозрастающая измеримая функиия, и пусть $\psi:[0,+\infty) \rightarrow \mathbb{R}-$ положительная непрерывная функиия. Пусть $\alpha>0, k_{0} \geqslant 1 u \sigma>0$. Предположим, что выполняются следующие условия:

(a) $\int_{1}^{+\infty} \frac{\varphi(s)}{s} d s<+\infty$;

(b) $s^{\alpha} \psi(s) \rightarrow+\infty$ npu $s \rightarrow+\infty$;

(c) если $k_{0} \leqslant s \leqslant t$, mo $s^{\alpha} \psi(s) \leqslant t^{\alpha} \psi(t)$;

(d) если $s>k_{0} u t=s[\psi(s)]^{1 / \alpha} \geqslant 1, \operatorname{mo} \varphi(s) \leqslant \sigma \varphi(t)$;

(e) $\forall k \geqslant k_{0} \quad \operatorname{meas}\{|v|>k\} \leqslant \frac{\varphi(k)}{k^{\alpha} \psi(k)}$.

Тогда $|v|^{\alpha} \psi(|v|) \in L^{1}(\Omega)$.

Д о к а з а т е л ь с т в о. Пусть $w: \Omega \rightarrow \mathbb{R}-$ функция такая, что

$$
w(x)= \begin{cases}k_{0}\left[\psi\left(k_{0}\right)\right]^{1 / \alpha}, & \text { если }|v(x)| \leqslant k_{0}, \\ |v(x)|[\psi(|v(x)|)]^{1 / \alpha}, & \text { если }|v(x)|>k_{0} .\end{cases}
$$

Ясно, что функция $w$ измерима. Покажем, что $w \in L^{\alpha}(\Omega)$. Положим $k_{*}=1+k_{0}\left[\psi\left(k_{0}\right)\right]^{1 / \alpha}$ и зафиксируем $k \geqslant k_{*}$. Легко видеть, что

$$
k_{0}^{\alpha} \psi\left(k_{0}\right)<k^{\alpha} .
$$

В силу условия (b) существует $k_{1}>k_{0}$ такое, что

$$
k_{1}^{\alpha} \psi\left(k_{1}\right)>k^{\alpha} .
$$


Ввиду непрерывности функции $\psi$ и неравенств (1.1) и $(1.2)$ существует $k_{2} \in\left(k_{0}, k_{1}\right)$ такое, что

$$
k_{2}^{\alpha} \psi\left(k_{2}\right)=k^{\alpha} .
$$

Тогда в силу условия (d) имеем

$$
\varphi\left(k_{2}\right) \leqslant \sigma \varphi(k) .
$$

Далее, пусть $x \in\{|w|>k\}$. Значит,

$$
w(x)>k .
$$

Поэтому из определения функции $w$ и неравенства $k \geqslant k_{*}$ следует, что $|v(x)|>k_{0}$. Тогда по определению функции $w$ имеем $w(x)=|v(x)|[\psi(|v(x)|)]^{1 / \alpha}$. Отсюда и из (1.5) вытекает неравенство

$$
|v(x)|^{\alpha} \psi(|v(x)|)>k^{\alpha} .
$$

Предположим, что $|v(x)| \leqslant k_{2}$. Тогда в силу условия (с) имеем $|v(x)|^{\alpha} \psi(|v(x)|) \leqslant k_{2}^{\alpha} \psi\left(k_{2}\right)$. Поэтому, учитывая (1.3), получим неравенство $|v(x)|^{\alpha} \psi(|v(x)|) \leqslant k^{\alpha}$, которое противоречит (1.6). Установленное противоречие доказывает, что $|v(x)|>k_{2}$. Значит, $x \in\left\{|v|>k_{2}\right\}$. Таким образом, $\{|w|>k\} \subset\left\{|v|>k_{2}\right\}$. Тогда

$$
\operatorname{meas}\{|w|>k\} \leqslant \operatorname{meas}\left\{|v|>k_{2}\right\} .
$$

Используя условие (е), равенство (1.3) и неравенство (1.4), получаем

$$
\operatorname{meas}\left\{|v|>k_{2}\right\} \leqslant \frac{\varphi\left(k_{2}\right)}{k_{2}^{\alpha} \psi\left(k_{2}\right)}=\frac{\varphi\left(k_{2}\right)}{k^{\alpha}} \leqslant \frac{\sigma \varphi(k)}{k^{\alpha}} .
$$

Отсюда и из (1.7) следует, что

$$
\operatorname{meas}\{|w|>k\} \leqslant \sigma k^{-\alpha} \varphi(k) .
$$

Итак, для любого $k \geqslant k_{*}$ справедливо неравенство (1.8). Теперь, учитывая условие (а), из предложения 1 выводим, что $w \in L^{\alpha}(\Omega)$. Поэтому $|v|^{\alpha} \psi(|v|) \in L^{1}(\Omega)$.

Формулировка следующей теоремы отличается от формулировки теоремы 1 тем, что условие (d) первой теоремы заменяется на требование ограниченности функции $\psi$.

Теорема 2. Пусть $v: \Omega \rightarrow \mathbb{R}-$ измеримая функиия, $\varphi:[1,+\infty) \rightarrow \mathbb{R}-$ неотрицательная невозрастающая измеримая функиия, и пусть $\psi:[0,+\infty) \rightarrow \mathbb{R}-$ положстельная ограниченная непрерывная функиия. Пусть $\alpha>0 u k_{0} \geqslant 1$. Предположим, что выполняются следуюшие условия:

(a) $\int_{1}^{+\infty} \frac{\varphi(s)}{s} d s<+\infty$;

(b) $s^{\alpha} \psi(s) \rightarrow+\infty$ npu $s \rightarrow+\infty$;

(c) если $k_{0} \leqslant s \leqslant t$, mo $s^{\alpha} \psi(s) \leqslant t^{\alpha} \psi(t)$;

(d) $\forall k \geqslant k_{0} \quad \operatorname{meas}\{|v|>k\} \leqslant \frac{\varphi(k)}{k^{\alpha} \psi(k)}$.

Тогда $|v|^{\alpha} \psi(|v|) \in L^{1}(\Omega)$.

Д о к а з а т е л ь с т в о. В силу ограниченности функции $\psi$ существует $c>0$ такое, что

$$
\forall s \in[0,+\infty) \quad \psi(s) \leqslant c .
$$

Положим

$$
\varphi_{1}=\frac{1}{c} \varphi, \quad \psi_{1}=\frac{1}{c} \psi
$$


Ясно, что функция $\varphi_{1}$ является неотрицательной, невозрастающей и измеримой, а функция $\psi_{1}$ положительна и непрерывна. В силу условия (а) имеем

$$
\int_{1}^{+\infty} \frac{\varphi_{1}(s)}{s} d s<+\infty
$$

а ввиду условий (b) и (с) справедливы следующие утверждения: $s^{\alpha} \psi_{1}(s) \rightarrow+\infty$ при $s \rightarrow+\infty$; если $k_{0} \leqslant s \leqslant t$, то $s^{\alpha} \psi_{1}(s) \leqslant t^{\alpha} \psi_{1}(t)$. Далее, пусть $s>k_{0}$ и $t=s\left[\psi_{1}(s)\right]^{1 / \alpha} \geqslant 1$. В силу (1.9) имеем $t \leqslant s$. Отсюда и из того, что функция $\varphi_{1}$ является невозрастающей, следует неравенство $\varphi_{1}(s) \leqslant \varphi_{1}(t)$. Наконец, в силу условия $(\mathrm{d})$ для любого $k \geqslant k_{0}$ имеем

$$
\operatorname{meas}\{|v|>k\} \leqslant \frac{\varphi_{1}(k)}{k^{\alpha} \psi_{1}(k)}
$$

Теперь из теоремы 1 выводим, что $|v|^{\alpha} \psi_{1}(|v|) \in L^{1}(\Omega)$. Поэтому $|v|^{\alpha} \psi(|v|) \in L^{1}(\Omega)$.

\section{2. Следствия}

Приведем следствия из общих теорем 1 и 2 для некоторых конкретных отношений функций $\varphi$ и $\psi$.

Следствие 1. Пусть $v: \Omega \rightarrow \mathbb{R}$ - измеримая функиия, $C>0, \alpha>0$, и пусть $\beta \in[0,1]$. Предположим, что

$$
\forall k \geqslant e \quad \operatorname{meas}\{|v|>k\} \leqslant C k^{-\alpha}(\ln k)^{-\beta} .
$$

Тогда для любого $\gamma>1-\beta$ имеем $|v|^{\alpha}[\ln (2+|v|)]^{-\gamma} \in L^{1}(\Omega)$.

Д о к а з а т е л ь с т в о. Зафиксируем $\gamma>1-\beta$, и пусть $\varphi:[1,+\infty) \rightarrow \mathbb{R}-$ функция такая, что

$$
\varphi(s)= \begin{cases}C, & \text { если } 1 \leqslant s<e, \\ C(\ln s)^{-\beta-\gamma}, & \text { если } s \geqslant e .\end{cases}
$$

Ясно, что функция $\varphi$ является неотрицательной, невозрастающей и измеримой.

Пусть $\psi:[0,+\infty) \rightarrow \mathbb{R}-$ функция такая, что

$$
\psi(s)= \begin{cases}1, & \text { если } 0 \leqslant s<e, \\ (\ln s)^{-\gamma}, & \text { если } s \geqslant e .\end{cases}
$$

Ясно, что функция $\psi$ положительна, ограничена и непрерывна.

Положим $k_{0}=\max \left\{e, e^{\gamma / \alpha}\right\}$ и покажем, что выполняются условия (a)-(d) теоремы 2.

Учитывая, что $\beta+\gamma>1$, для произвольного $N>e$ получаем

$$
\int_{e}^{N} \frac{\varphi(s)}{s} d s=\left.\frac{C}{1-\beta-\gamma}(\ln s)^{1-\beta-\gamma}\right|_{e} ^{N} \leqslant \frac{C}{\beta+\gamma-1} .
$$

Отсюда следует, что

$$
\int_{1}^{+\infty} \frac{\varphi(s)}{s} d s<+\infty
$$

Далее, заметим, что для любых $\lambda, s>0$ справедливо неравенство $\lambda \ln s<s^{\lambda}$. Используя это, для произвольного $s \geqslant e$ получаем $s^{\alpha} \psi(s)=s^{\alpha}(\ln s)^{-\gamma} \geqslant\left(\frac{\alpha}{2 \gamma}\right)^{\gamma} s^{\alpha / 2}$. Поэтому $s^{\alpha} \psi(s) \rightarrow+\infty$ при $s \rightarrow+\infty$. 
Пусть $h:(1,+\infty) \rightarrow \mathbb{R}-$ функция такая, что $h(s)=s^{\alpha}(\ln s)^{-\gamma}$ для любого $s \in(1,+\infty)$. Имеем $h^{\prime}(s)=s^{\alpha-1}(\ln s)^{-\gamma-1}(\alpha \ln s-\gamma)$ для любого $s \in(1,+\infty)$. Отсюда следует, что $h^{\prime} \geqslant 0$ на $\left[k_{0},+\infty\right)$. Поэтому если $k_{0} \leqslant s \leqslant t$, то $s^{\alpha} \psi(s) \leqslant t^{\alpha} \psi(t)$.

Наконец, в силу $(2.1)$ и определения функций $\varphi$ и $\psi$ для любого $k \geqslant k_{0}$ справедливо неравенство

$$
\operatorname{meas}\{|v|>k\} \leqslant \frac{\varphi(k)}{k^{\alpha} \psi(k)} .
$$

Таким образом, условия (a)-(d) теоремы 2 выполняются. Поэтому в силу этой теоремы $|v|^{\alpha} \psi(|v|) \in L^{1}(\Omega)$. Следовательно, $|v|^{\alpha}[\ln (2+|v|)]^{-\gamma} \in L^{1}(\Omega)$.

Следствие 2. Пусть $v: \Omega \rightarrow \mathbb{R}$ - измеримая функиия, $C>0, \alpha>0$, и пусть $\beta>1$. Предположим, что

$$
\forall k \geqslant e \quad \operatorname{meas}\{|v|>k\} \leqslant C k^{-\alpha}(\ln k)^{-\beta} .
$$

Тогда для любого $\gamma \in(0, \beta-1)$ имеем $|v|^{\alpha}[\ln (1+|v|)]^{\gamma} \in L^{1}(\Omega)$.

Д о к а з а т е л ь с т в о. Зафиксируем $\gamma \in(0, \beta-1)$, и пусть $\varphi:[1,+\infty) \rightarrow \mathbb{R}-$ функция такая, что

$$
\varphi(s)= \begin{cases}C, & \text { если } 1 \leqslant s<e, \\ C(\ln s)^{\gamma-\beta}, & \text { если } s \geqslant e .\end{cases}
$$

Ясно, что функция $\varphi$ является неотрицательной, невозрастающей и измеримой.

Пусть $\psi:[0,+\infty) \rightarrow \mathbb{R}-$ функция такая, что

$$
\psi(s)= \begin{cases}1, & \text { если } 0 \leqslant s<e, \\ (\ln s)^{\gamma}, & \text { если } s \geqslant e .\end{cases}
$$

Ясно, что функция $\psi$ положительна и непрерывна.

Положим $k_{0}=e$ и $\sigma=(1+\gamma / \alpha)^{\beta-\gamma}$. Теперь заметим, что выполняются условия (a)-(e) теоремы 1. Действительно, условие (а) теоремы 1 выполняется в силу неравенства $\beta-\gamma>1$. Выполнение условий (b) и (с) теоремы 1 очевидно. Далее, пусть $s>k_{0}$ и $t=s[\psi(s)]^{1 / \alpha} \geqslant 1$. Следовательно, $t^{\alpha}=s^{\alpha}(\ln s)^{\gamma} \leqslant s^{\alpha+\gamma}$. Тогда $\alpha \ln t \leqslant(\alpha+\gamma) \ln s$. Отсюда вытекает, что $(\ln s)^{\gamma-\beta} \leqslant(1+\gamma / \alpha)^{\beta-\gamma}(\ln t)^{\gamma-\beta}$. Значит, $\varphi(s) \leqslant \sigma \varphi(t)$. Таким образом, выполняется условие $(\mathrm{d})$ теоремы 1 . Наконец, в силу $(2.2)$ и определения функций $\varphi$ и $\psi$ выполняется условие (е) теоремы 1. Теперь из этой теоремы выводим, что $|v|^{\alpha} \psi(|v|) \in L^{1}(\Omega)$. Следовательно, $|v|^{\alpha}[\ln (1+|v|)]^{\gamma} \in L^{1}(\Omega)$.

Заметим, что если выполняются условия следствия 2 , то согласно доказанной в [3] лемме 2 с равносильными условиями имеем только включение $v \in L^{\alpha}(\Omega)$. Таким образом, утверждение следствия 2 сильнее утверждения упомянутой леммы из [3].

Следствие 3. Пусть $v: \Omega \rightarrow \mathbb{R}$ - измеримая функиия, $C>0, \alpha>0$, и пусть $\beta \in[0,1]$. Предположсим, что

$$
\forall k \geqslant 3 \quad \operatorname{meas}\{|v|>k\} \leqslant C k^{-\alpha}(\ln \ln k)^{-\beta} .
$$

Тогда для любого $\gamma>1-\beta$ имеем $|v|^{\alpha}[\ln (2+|v|)]^{-1}[\ln \ln (3+|v|)]^{-\gamma} \in L^{1}(\Omega)$.

Д о к а з а т е л ь с т в о. Зафиксируем $\gamma>1-\beta$, и пусть $\varphi:[1,+\infty) \rightarrow \mathbb{R}-$ функция такая, что

$$
\varphi(s)=\left\{\begin{array}{l}
C(\ln 3)^{-1}(\ln \ln 3)^{-\beta-\gamma}, \text { если } 1 \leqslant s<3, \\
C(\ln s)^{-1}(\ln \ln s)^{-\beta-\gamma}, \text { если } s \geqslant 3 .
\end{array}\right.
$$

Ясно, что функция $\varphi$ является неотрицательной, невозрастающей и измеримой. 
Пусть $\psi:[0,+\infty) \rightarrow \mathbb{R}-$ функция такая, что

$$
\psi(s)=\left\{\begin{array}{l}
(\ln 3)^{-1}(\ln \ln 3)^{-\gamma}, \text { если } 0 \leqslant s<3, \\
(\ln s)^{-1}(\ln \ln s)^{-\gamma}, \text { если } s \geqslant 3 .
\end{array}\right.
$$

Ясно, что функция $\psi$ положительна, ограничена и непрерывна.

Положим $k_{0}=\max \left\{e^{e}, e^{(1+\gamma) / \alpha}\right\}$. Аналогично доказательству следствия 1 с учетом $(2.3)$ устанавливаем, что выполняются условия (a)-(d) теоремы 2. Поэтому в силу этой теоремы $|v|^{\alpha} \psi(|v|) \in L^{1}(\Omega)$. Следовательно, $|v|^{\alpha}[\ln (2+|v|)]^{-1}[\ln \ln (3+|v|)]^{-\gamma} \in L^{1}(\Omega)$.

Следствие 4. Пусть $v: \Omega \rightarrow \mathbb{R}-$ измеримая функиия, $C>0, \alpha>0$, и пусть $\beta>1$. Предположим, что

$$
\forall k \geqslant 3 \quad \operatorname{meas}\{|v|>k\} \leqslant C k^{-\alpha}(\ln \ln k)^{-\beta} .
$$

Тогда для любого $\gamma \in(0, \beta-1)$ имеем $|v|^{\alpha}[\ln (2+|v|)]^{-1}[\ln \ln (3+|v|)]^{\gamma} \in L^{1}(\Omega)$.

Д о к а з а т е л ь с т в о. Зафиксируем $\gamma \in(0, \beta-1)$, и пусть $\varphi:[1,+\infty) \rightarrow \mathbb{R}-$ функция такая, что

$$
\varphi(s)=\left\{\begin{array}{l}
C(\ln 3)^{-1}(\ln \ln 3)^{\gamma-\beta}, \text { если } 1 \leqslant s<3, \\
C(\ln s)^{-1}(\ln \ln s)^{\gamma-\beta}, \text { если } s \geqslant 3 .
\end{array}\right.
$$

Ясно, что функция $\varphi$ является неотрицательной, невозрастающей и измеримой.

Пусть $\psi:[0,+\infty) \rightarrow \mathbb{R}-$ функция такая, что

$$
\psi(s)= \begin{cases}(\ln 3)^{-1}(\ln \ln 3)^{\gamma} & \text { если } 0 \leqslant s<3, \\ (\ln s)^{-1}(\ln \ln s)^{\gamma}, & \text { если } s \geqslant 3 .\end{cases}
$$

Ясно, что функция $\psi$ положительна, ограничена и непрерывна.

Положим $k_{0}=\max \left\{3, e^{1 / \alpha}\right\}$. Аналогично доказательству следствия 1 с учетом (2.4) устанавливаем, что выполняются условия (a) $-(\mathrm{d})$ теоремы 2 . Поэтому в силу этой теоремы имеем $|v|^{\alpha} \psi(|v|) \in L^{1}(\Omega)$. Следовательно, $|v|^{\alpha}[\ln (2+|v|)]^{-1}[\ln \ln (3+|v|)]^{\gamma} \in L^{1}(\Omega)$.

Далее, введем следующие классы функций. Для любых $\alpha>0$ и $\gamma \geqslant 0$ положим

$$
\begin{gathered}
K_{1,-}^{\alpha, \gamma}(\Omega)=\left\{v: \Omega \rightarrow \mathbb{R}: v \text { измерима и }|v|^{\alpha}[\ln (2+|v|)]^{-\gamma} \in L^{1}(\Omega)\right\}, \\
K_{2,-}^{\alpha, \gamma}(\Omega)=\left\{v: \Omega \rightarrow \mathbb{R}: v \text { измерима и }|v|^{\alpha}[\ln (2+|v|)]^{-1}[\ln \ln (3+|v|)]^{-\gamma} \in L^{1}(\Omega)\right\} .
\end{gathered}
$$

Для любых $\alpha>0$ и $\gamma>0$ положим

$$
\begin{gathered}
K_{1,+}^{\alpha, \gamma}(\Omega)=\left\{v: \Omega \rightarrow \mathbb{R}: v \text { измерима и }|v|^{\alpha}[\ln (1+|v|)]^{\gamma} \in L^{1}(\Omega)\right\}, \\
K_{2,+}^{\alpha, \gamma}(\Omega)=\left\{v: \Omega \rightarrow \mathbb{R}: v \text { измерима и }|v|^{\alpha}[\ln (2+|v|)]^{-1}[\ln \ln (3+|v|)]^{\gamma} \in L^{1}(\Omega)\right\} .
\end{gathered}
$$

При любом фиксированном $\alpha>0$ эти классы функций близки к пространству $L^{\alpha}(\Omega)$ в смысле, указанном во введении. Используя их, результаты, полученные в данном разделе, можно выразить следующим образом:

(i) если выполняются условия следствия 1 , то $v \in K_{1,-}^{\alpha, \gamma}(\Omega)$ для любого $\gamma>1-\beta$;

(ii) если выполняются условия следствия 2 , то $v \in K_{1,+}^{\alpha, \gamma}(\Omega)$ для любого $\gamma \in(0, \beta-1)$;

(iii) если выполняются условия следствия 3 , то $v \in K_{2,-}^{\alpha, \gamma}(\Omega)$ для любого $\gamma>1-\beta$;

(iv) если выполняются условия следствия 4 , то $v \in K_{2,+}^{\alpha, \gamma}(\Omega)$ для любого $\gamma \in(0, \beta-1)$. 


\section{3. Примеры}

Рассмотрим примеры, показывающие точность результатов предыдущего раздела в соответствующих шкалах классов, введенных выше.

П р и м е р 1. Пусть $\Omega=\left\{x \in \mathbb{R}^{n}:|x|<1 / e\right\}, \alpha>0$, и пусть $\beta \geqslant 0$. Пусть $v: \Omega \rightarrow \mathbb{R}-$ функция такая, что

$$
v(x)= \begin{cases}|x|^{-n / \alpha}\left(\ln \frac{1}{|x|}\right)^{-\beta / \alpha}, & \text { если } x \in \Omega \backslash\{0\}, \\ 0, & \text { если } x=0 .\end{cases}
$$

Ясно, что функция $v$ измерима. Зафиксируем $k \geqslant e$, и пусть $x \in\{|v|>k\}$. Ясно, что $x \in \Omega \backslash\{0\}$. В силу указанных включений и определения функции $v$ имеем

$$
|x|^{-n / \alpha}\left(\ln \frac{1}{|x|}\right)^{-\beta / \alpha}>k, \quad \ln \frac{1}{|x|}>1 .
$$

Отсюда выводим, что $|x|^{-n / \alpha}>k$. Тогда $\ln k<(n / \alpha) \ln (1 /|x|)$. Используя это неравенство и первое из неравенств (3.1), находим, что $|x|^{n}<(n / \alpha)^{\beta} k^{-\alpha}(\ln k)^{-\beta}$. Поэтому ввиду произвольности $x \in\{|v|>k\}$ множество $\{|v|>k\}$ содержится в $n$-мерном шаре с центром в нуле и радиусом $(n / \alpha)^{\beta / n} k^{-\alpha / n}(\ln k)^{-\beta / n}$. Следовательно, в силу произвольности $k \geqslant e$ заключаем, что

$$
\forall k \geqslant e \quad \operatorname{meas}\{|v|>k\} \leqslant \omega_{n}(n / \alpha)^{\beta} k^{-\alpha}(\ln k)^{-\beta},
$$

где $\omega_{n}$ - мера единичного шара в $\mathbb{R}^{n}$.

Далее, пусть $w: \Omega \rightarrow \mathbb{R}-$ функция такая, что

$$
w(x)= \begin{cases}|x|^{-n}\left(\ln \frac{1}{|x|}\right)^{-1}, & \text { если } x \in \Omega \backslash\{0\}, \\ 0, & \text { если } x=0 .\end{cases}
$$

Легко видеть, что $w \notin L^{1}(\Omega)$.

Предположим, что $\beta \in[0,1]$. Учитывая (3.2), из следствия 1 выводим, что для любого $\gamma>1-\beta$ справедливо включение $|v|^{\alpha}[\ln (2+|v|)]^{-\gamma} \in L^{1}(\Omega)$. Однако если $\gamma=1-\beta$, то $|v|^{\alpha}[\ln (2+|v|)]^{-\gamma} \notin L^{1}(\Omega)$. Действительно, пусть $x \in \Omega \backslash\{0\}$, причем $|x| \leqslant(1 / e)^{\alpha /(n-1)}$. Имеем $|x|^{-(n-1) / \alpha} \geqslant e$ и, следовательно, $|v(x)| \geqslant e$. Тогда $\ln (2+|v(x)|) \leqslant 2 \ln |v(x)|$. Кроме того, в силу определения функции $v$ имеем $\ln |v(x)| \leqslant(n / \alpha) \ln (1 /|x|)$. Из последних двух неравенств следует, что $w(x) \leqslant(2 n / \alpha)^{1-\beta}|v(x)|^{\alpha}[\ln (2+|v(x)|)]^{-(1-\beta)}$. Полученная оценка и свойство $w \notin L^{1}(\Omega)$ приводят к заключению, что $|v|^{\alpha}[\ln (2+|v|)]^{-(1-\beta)} \notin L^{1}(\Omega)$. Установленный результат показывает, что в формулировке следствия 1 неравенство $\gamma>1-\beta$ нельзя заменить на неравенство $\gamma \geqslant 1-\beta$ без нарушения результирующего включения.

Теперь пусть $\beta>1$. Учитывая (3.2), из следствия 2 выводим, что для любого $\gamma \in(0, \beta-1)$ справедливо включение $|v|^{\alpha}[\ln (1+|v|)]^{\gamma} \in L^{1}(\Omega)$. Вместе с тем $|v|^{\alpha}[\ln (1+|v|)]^{\beta-1} \notin L^{1}(\Omega)$. Действительно, пусть $x \in \mathbb{R}^{n}$, причем $0<|x| \leqslant e^{-4 \beta^{2}}$. Имеем $\ln (1 /|x|) \geqslant 4 \beta^{2}$ и, следовательно, $\ln \ln (1 /|x|) \leqslant(1 / \beta) \ln (1 /|x|)$. Тогда $\ln |v(x)|=(n / \alpha) \ln (1 /|x|)-(\beta / \alpha) \ln \ln (1 /|x|) \geqslant$ $(1 / \alpha) \ln (1 /|x|)$. Следовательно, $w(x) \leqslant \alpha^{\beta-1}|v(x)|^{\alpha}[\ln (1+|v(x)|)]^{\beta-1}$. Полученная оценка и свойство $w \notin L^{1}(\Omega)$ приводят к заключению, что $|v|^{\alpha}[\ln (1+|v|)]^{\beta-1} \notin L^{1}(\Omega)$. Установленный результат показывает, что в формулировке следствия 2 включение $\gamma \in(0, \beta-1)$ нельзя заменить на включение $\gamma \in(0, \beta-1]$ без нарушения результирующего включения.

Рассмотренный пример показывает, что утверждение следствия 1 является точным в шкале классов $K_{1,-}^{\alpha, \gamma}(\Omega), \gamma \geqslant 0$, а утверждение следствия 2 является точным в шкале классов $K_{1,+}^{\alpha, \gamma}(\Omega)$, $\gamma>0$. 
П р и м е р 2. Пусть $\Omega=\left\{x \in \mathbb{R}^{n}:|x|<1 / e^{3}\right\}, \alpha>0$, и пусть $\beta \geqslant 0$. Пусть $v: \Omega \rightarrow \mathbb{R}$ функция такая, что

$$
v(x)= \begin{cases}|x|^{-n / \alpha}\left(\ln \ln \frac{1}{|x|}\right)^{-\beta / \alpha}, & \text { если } x \in \Omega \backslash\{0\}, \\ 0, & \text { если } x=0 .\end{cases}
$$

Ясно, что функция $v$ измерима. Зафиксируем $k>e^{3}$, и пусть $x \in\{|v|>k\}$. Ясно, что $x \in$ $\Omega \backslash\{0\}$. В силу указанных включений и определения функции $v$ имеем

$$
|x|^{-n / \alpha}\left(\ln \ln \frac{1}{|x|}\right)^{-\beta / \alpha}>k, \quad \ln \ln \frac{1}{|x|}>1 .
$$

Отсюда выводим, что $|x|^{-n / \alpha}>k$. Тогда $\ln \ln k<(1+|\ln (n / \alpha)|) \ln \ln (1 /|x|)$. Используя это неравенство и первое из неравенств (3.3), находим, что $|x|^{n}<(1+|\ln (n / \alpha)|)^{\beta} k^{-\alpha}(\ln \ln k)^{-\beta}$. Из полученной оценки вытекает неравенство meas $\{|v|>k\} \leqslant \omega_{n}(1+|\ln (n / \alpha)|)^{\beta} k^{-\alpha}(\ln \ln k)^{-\beta}$. В случае $3 \leqslant k \leqslant e^{3}$, не обращаясь к определению функции $v$, получаем meas $\{|v|>k\} \leqslant$ meas $\Omega=\omega_{n} e^{-3 n} \leqslant \omega_{n} e^{3(\alpha-n)}(\ln 3)^{\beta} k^{-\alpha}(\ln \ln k)^{-\beta}$. В силу изложенного заключаем, что

$$
\forall k \geqslant 3 \quad \operatorname{meas}\{|v|>k\} \leqslant \omega_{n} e^{3 \alpha}(\ln 3+|\ln (n / \alpha)|)^{\beta} k^{-\alpha}(\ln \ln k)^{-\beta} .
$$

Далее, пусть $w: \Omega \rightarrow \mathbb{R}-$ функция такая, что

$$
w(x)= \begin{cases}|x|^{-n}\left(\ln \frac{1}{|x|}\right)^{-1}\left(\ln \ln \frac{1}{|x|}\right)^{-1}, & \text { если } x \in \Omega \backslash\{0\}, \\ 0, & \text { если } x=0 .\end{cases}
$$

Легко видеть, что $w \notin L^{1}(\Omega)$.

Предположим, что $\beta \in[0,1]$. Учитывая (3.4), из следствия 3 выводим, что для любого $\gamma>1-\beta$ справедливо включение $|v|^{\alpha}[\ln (2+|v|)]^{-1}[\ln \ln (3+|v|)]^{-\gamma} \in L^{1}(\Omega)$. Однако если $\gamma=1-\beta$, то $|v|^{\alpha}[\ln (2+|v|)]^{-1}[\ln \ln (3+|v|)]^{-\gamma} \notin L^{1}(\Omega)$. Действительно, пусть $x \in \Omega \backslash\{0\}$, причем $|x| \leqslant e^{-\alpha e /(n-1)}$. Поскольку $\beta \leqslant 1$ и $\ln \ln (1 /|x|)<1 /|x|$, в силу определения функции $v$ имеем $|v(x)| \geqslant|x|^{-(n-1) / \alpha} \geqslant e^{e}$. Следовательно, $\ln (2+|v(x)|) \leqslant 2 \ln |v(x)|$ и $\ln \ln (3+|v(x)|) \leqslant$ $2 \ln \ln |v(x)|$. Учитывая эти неравенства, получим

$$
|v(x)|^{\alpha}[\ln |v(x)|]^{-1}[\ln \ln |v(x)|]^{-(1-\beta)} \leqslant 4|v(x)|^{\alpha}[\ln (2+|v(x)|)]^{-1}[\ln \ln (3+|v(x)|)]^{-(1-\beta)} .
$$

Кроме того, поскольку $0<|x|<1 / e^{3}$, имеем $\ln \ln (1 /|x|)>1$. Поэтому из определения функции $v$ следует, что $\ln |v(x)| \leqslant(n / \alpha) \ln (1 /|x|)$ и $\ln \ln |v(x)| \leqslant(1+|\ln (n / \alpha)|) \ln \ln (1 /|x|)$. Используя эти неравенства, находим, что $w(x) \leqslant(n / \alpha)(1+|\ln (n / \alpha)|)|v(x)|^{\alpha}[\ln |v(x)|]^{-1}[\ln \ln |v(x)|]^{-(1-\beta)}$. Отсюда и из (3.5) выводим неравенство

$$
w(x) \leqslant(4 n / \alpha)(1+|\ln (n / \alpha)|)|v(x)|^{\alpha}[\ln (2+|v(x)|)]^{-1}[\ln \ln (3+|v(x)|)]^{-(1-\beta)} .
$$

Полученная оценка вместе со свойством $w \notin L^{1}(\Omega)$ приводит к заключению, что функция $|v|^{\alpha}[\ln (2+|v|)]^{-1}[\ln \ln (3+|v|)]^{-(1-\beta)}$ не принадлежит $L^{1}(\Omega)$. Установленный результат показывает, что в формулировке следствия 3 неравенство $\gamma>1-\beta$ нельзя заменить на неравенство $\gamma \geqslant 1-\beta$ без нарушения результирующего включения.

Теперь пусть $\beta>1$. Учитывая (3.4), из следствия 4 выводим, что для любого $\gamma \in(0, \beta-1)$ справедливо включение $|v|^{\alpha}[\ln (2+|v|)]^{-1}[\ln \ln (3+|v|)]^{\gamma} \in L^{1}(\Omega)$. Однако если $\gamma=\beta-1$, то последнее включение неверно. Действительно, положим $\lambda=\max \left\{4 \beta^{2}, \alpha e\right\}$, и пусть $x \in \mathbb{R}^{n}$, причем $0<|x| \leqslant e^{-\lambda}$. Имеем $\ln (1 /|x|) \geqslant 4 \beta^{2}$ и, следовательно, $\ln \ln \ln (1 /|x|) \leqslant(1 / \beta) \ln (1 /|x|)$. Тогда в силу определения функции $v$ и неравенства $|x| \leqslant e^{-\alpha e}$ имеем

$$
\ln |v(x)|=\frac{n}{\alpha} \ln \frac{1}{|x|}-\frac{\beta}{\alpha} \ln \ln \ln \frac{1}{|x|} \geqslant \frac{1}{\alpha} \ln \frac{1}{|x|} \geqslant e .
$$


Поэтому

$$
\ln \ln \frac{1}{|x|} \leqslant(1+|\ln \alpha|) \ln \ln |v(x)|, \quad \ln (2+|v(x)|) \leqslant 2 \ln |v(x)| .
$$

Кроме того, в силу определения функции $v$ и неравенства $\ln \ln (1 /|x|)>1$ имеем $|v(x)| \leqslant$ $|x|^{-n / \alpha}$ и, следовательно, $\ln |v(x)| \leqslant(n / \alpha) \ln (1 /|x|)$. Отсюда и из неравенств (3.6) выводим, что $w(x) \leqslant(2 n / \alpha)(1+|\ln \alpha|)^{\beta-1}|v(x)|^{\alpha}[\ln (2+|v(x)|)]^{-1}[\ln \ln (3+|v(x)|)]^{\beta-1}$. Полученная оценка и свойство $w \notin L^{1}(\Omega)$ приводят к заключению, что $|v|^{\alpha}[\ln (2+|v|)]^{-1}[\ln \ln (3+|v|)]^{\beta-1} \notin L^{1}(\Omega)$. Установленный результат показывает, что в формулировке следствия 4 включение $\gamma \in(0, \beta-1)$ нельзя заменить на включение $\gamma \in(0, \beta-1]$ без нарушения результирующего включения.

Рассмотренный пример показывает, что утверждение следствия 3 является точным в шкале классов $K_{2,-}^{\alpha, \gamma}(\Omega), \gamma \geqslant 0$, а утверждение следствия 4 является точным в шкале классов $K_{2,+}^{\alpha, \gamma}(\Omega)$, $\gamma>0$.

Заметим, что несмотря на установленную точность утверждений следствий 1-4 в указанных шкалах классов функций, эти утверждения можно усилить. Например, если выполняются условия следствия 2 , то для любого $\lambda>1$ имеем $|v|^{\alpha}[\ln (1+|v|)]^{\beta-1}[\ln \ln (3+|v|)]^{-\lambda} \in L^{1}(\Omega)$.

\section{4. Приложения}

Можно указать целый ряд приложений результатов разд. 1 и 2 к исследованию свойств интегрируемости решений эллиптических уравнений и вариационных неравенств с правой частью из классов функций, близких к $L^{1}(\Omega)$. В этом разделе ограничимся несколькими приложениями результатов разд. 2 к энтропийным и слабым решениям задачи Дирихле для нелинейных эллиптических уравнений второго порядка с правой частью из некоторых классов, близких к $L^{1}(\Omega)$ и определяемых с помощью логарифмической функции или ее двукратной композиции. Другие приложения результатов настоящей работы будут даны в последующих публикациях автора.

Пусть $p \in(1, n)$. Пусть $c_{1}, c_{2}>0, g \in L^{p /(p-1)}(\Omega), g \geqslant 0$ в $\Omega$, и пусть $a_{i}: \Omega \times \mathbb{R}^{n} \rightarrow \mathbb{R}-$ функция Каратеодори для любого $i \in\{1, \ldots, n\}$. Предположим, что для почти всех $x \in \Omega$ и любого $\xi \in \mathbb{R}^{n}$

$$
\sum_{i=1}^{n}\left|a_{i}(x, \xi)\right| \leqslant c_{1}|\xi|^{p-1}+g(x), \quad \sum_{i=1}^{n} a_{i}(x, \xi) \xi_{i} \geqslant c_{2}|\xi|^{p}
$$

Кроме того, предположим, что для почти всех $x \in \Omega$ и любых $\xi, \xi^{\prime} \in \mathbb{R}^{n}, \xi \neq \xi^{\prime}$,

$$
\sum_{i=1}^{n}\left[a_{i}(x, \xi)-a_{i}\left(x, \xi^{\prime}\right)\right]\left(\xi_{i}-\xi_{i}^{\prime}\right)>0 .
$$

Пусть $f \in L^{1}(\Omega)$. Рассмотрим следующую задачу Дирихле:

$$
-\sum_{i=1}^{n} \frac{\partial}{\partial x_{i}} a_{i}(x, \nabla u)=f \text { в } \Omega, \quad u=0 \text { на } \partial \Omega .
$$

О п р е д е л е н и е 1 . Слабое решение задачи (4.1) есть функция $u \in \stackrel{\circ}{W^{1,1}}(\Omega)$ такая, что:

(i) для любого $i \in\{1, \ldots, n\}$ имеем $a_{i}(x, \nabla u) \in L^{1}(\Omega)$;

(ii) для любой функции $v \in C_{0}^{\infty}(\Omega)$

$$
\int_{\Omega}\left\{\sum_{i=1}^{n} a_{i}(x, \nabla u) D_{i} v\right\} d x=\int_{\Omega} f v d x .
$$

Заметим, что если $p>2-1 / n$, то согласно теореме 1 из [5] существует слабое решение задачи (4.1), принадлежащее $\stackrel{\circ}{W}{ }^{1, \lambda}(\Omega)$ для любого $\lambda, 1 \leqslant \lambda<n(p-1) /(n-1)$. 
Далее, пусть для любого $k>0$ функция $T_{k}: \mathbb{R} \rightarrow \mathbb{R}$ определена следующим образом:

$$
T_{k}(s)= \begin{cases}s, & \text { если }|s| \leqslant k, \\ k \operatorname{sign} s, & \text { если }|s|>k .\end{cases}
$$

Обозначим через $\stackrel{\circ}{\mathcal{T}}^{1, p}(\Omega)$ множество всех функций $v: \Omega \rightarrow \mathbb{R}$ таких, что $T_{k}(v) \in \stackrel{\circ}{W}^{1, p}(\Omega)$ для любого $k>0$. Заметим, что $\stackrel{\circ}{W}^{1, p}(\Omega) \subset \stackrel{\circ}{\mathcal{T}}^{1, p}(\Omega)$ и множество $\stackrel{\circ}{\mathcal{T}} 1, p(\Omega) \backslash L^{1}(\Omega)$ непусто. Для произвольных $v: \Omega \rightarrow \mathbb{R}$ и $x \in \Omega$ положим $k(v, x)=\min \{l \in \mathbb{N}:|v(x)| \leqslant l\}$.

О п р е д е л е н и е 2. Пусть $v \in \stackrel{\circ}{\mathcal{T}}^{1, p}(\Omega)$ и $i \in\{1, \ldots, n\}$. Тогда $\delta_{i} v-$ функция на $\Omega$ такая, что $\delta_{i} v(x)=D_{i} T_{k(v, x)}(v)(x)$ для любого $x \in \Omega$.

Заметим, что если $v \in \stackrel{\circ}{\mathcal{T}}^{1, p}(\Omega)$ и $i \in\{1, \ldots, n\}$, то для любого $k>0$ имеем $D_{i} T_{k}(v)=$ $\delta_{i} v \cdot 1_{\{|v|<k\}}$ п.в. в $\Omega$ (см. [4, предложение 1.3]). Поэтому если $v \in \stackrel{\circ}{W^{1, p}}(\Omega)$, то для любого $i \in\{1, \ldots, n\}$ имеем $\delta_{i} v=D_{i} v$ п.в. в $\Omega$.

О п р е д е л е н и е 3. Если $v \in \stackrel{\circ}{\mathcal{T}}^{1, p}(\Omega)$, то $\delta v$ - отображение $\Omega$ в $\mathbb{R}^{n}$ такое, что для любых $x \in \Omega$ и $i \in\{1, \ldots, n\}$ имеем $(\delta v(x))_{i}=\delta_{i} v(x)$.

Заметим, что если $u \in \stackrel{\circ}{\mathcal{T}}^{1, p}(\Omega), v \in \stackrel{\circ}{W}^{1, p}(\Omega) \cap L^{\infty}(\Omega), k>0$ и $i \in\{1, \ldots, n\}$, то функция $a_{i}(x, \delta u)\left(\delta_{i} u-\delta_{i} v\right)$ суммируема на множестве $\{|u-v|<k\}$.

О п р е д е л е н и е 4. Энтропийное решение задачи (4.1) есть функция $u \in \stackrel{\circ}{\mathcal{T}}^{1, p}(\Omega)$ такая, что для любых $v \in C_{0}^{\infty}(\Omega)$ и $k>0$

$$
\int_{\{|u-v|<k\}}\left\{\sum_{i=1}^{n} a_{i}(x, \delta u)\left(\delta_{i} u-\delta_{i} v\right)\right\} d x \leqslant \int_{\Omega} f T_{k}(u-v) d x .
$$

В силу теоремы 6.1 из [2] существует единственное энтропийное решение задачи (4.1). Заметим также, что если $p>2-1 / n$ и $u$ - энтропийное решение задачи (4.1), то $u-$ слабое решение этой задачи (см., например, [2;4]).

Положим $q=n(p-1) /(n-p)$. В силу лемм 3.1 и 4.1 из работы [2] энтропийное решение задачи (4.1) принадлежит $L^{\lambda}(\Omega)$ для любого $\lambda \in(0, q)$. Вместе с тем энтропийное решение задачи (4.1), вообще говоря, не принадлежит пространству $L^{q}(\Omega)$. По этому поводу см., в частности, [6, пример 1.4.2]. В случае, когда $p \geqslant 2-1 / n$ и $f \ln (1+|f|) \in L^{1}(\Omega)$, существование слабого решения задачи (4.1), принадлежащего $L^{q}(\Omega)$, следует из результатов работ [5; 7]. Более сильные утверждения относительно решений задачи (4.1) вытекают из результатов, установленных в [4]. В частности, в силу теоремы 3.1 из [4] энтропийное решение задачи (4.1) принадлежит $L^{q}(\Omega)$, если при некотором $\lambda>(n-p) / n$

$$
f[\ln (1+|f|)]^{\lambda} \in L^{1}(\Omega) .
$$

Кроме того, из той же теоремы следует, что если $p>2-1 / n$ и при некотором $\lambda>(n-p) / n$ справедливо включение (4.2), то существует слабое решение задачи (4.1), принадлежащее $L^{q}(\Omega)$. Если же $p=2-1 / n$ и при некотором $\lambda>(n-1) / n$ справедливо включение $(4.2)$, то существование слабого решения задачи (4.1), принадлежащего $L^{q}(\Omega)$, устанавливается с помощью теоремы 3.2 из [4]. Ниже покажем, что выполнение включения (4.2) с $\lambda>(n-p) / n$ обеспечивает более сильную интегрируемость решений задачи (4.1) по сравнению с $L^{q}$-интегрируемостью. Это будет следствием рассмотрения общего случая, когда $f$ удовлетворяет включению (4.2) с $\lambda>0$. Изучим также случай, когда $f[\ln \ln (e+|f|)]^{\lambda} \in L^{1}(\Omega)$ с $\lambda>0$. Для этих целей понадобится следующий вспомогательный результат. 
Предложение 2. Пусть $u$ - энтропийное решение задачи (4.1). Пусть $\gamma>0 u k>0$. Тогда

$$
\operatorname{meas}\{|u| \geqslant k\} \leqslant c k^{-q}\left(k^{\gamma p /(p-1)-1}+\int_{\left\{|f| \geqslant k^{\gamma}\right\}}|f| d x\right)^{n /(n-p)},
$$

где с - положительная константа, зависящал только от $n, p$, meas $\Omega u c_{2}$.

Это предложение является следствием леммы 2.3 из [4].

Перейдем к результатам, вытекающим из следствий 1-4 и предложения 2.

Предложение 3. Пусть и - энтропийное решение задачи (4.1). Тогда для любого $\gamma>1$ имеем $|u|^{q}[\ln (2+|u|)]^{-1}[\ln \ln (3+|u|)]^{-\gamma} \in L^{1}(\Omega)$.

Д о к а з а т е л ь с т в о. Положим $\beta=0$, и пусть $k \geqslant 3$. В силу предложения 2 справедливо неравенство meas $\{|u|>k\} \leqslant c\left(1+\|f\|_{L^{1}(\Omega)}\right)^{n /(n-p)} k^{-q}(\ln \ln k)^{-\beta}$. Поэтому ввиду следствия 3 для любого $\gamma>1$ имеем $|u|^{q}[\ln (2+|u|)]^{-1}[\ln \ln (3+|u|)]^{-\gamma} \in L^{1}(\Omega)$.

Учитывая, что при $p>2-1 / n$ энтропийное решение задачи (4.1) является слабым решением этой задачи, из предложения 3 выводим следующий результат.

Предложение 4. Пусть $p>2-1 / n$. Тогда существует слабое решение и задачи (4.1) такое, что для любого $\gamma>1$ имеем $|u|^{q}[\ln (2+|u|)]^{-1}[\ln \ln (3+|u|)]^{-\gamma} \in L^{1}(\Omega)$.

Заметим, что теорема 2.1 из [8] дает существование слабого решения $u$ задачи (4.1) такого, что $|u|^{q}[\ln (2+|u|)]^{-\gamma} \in L^{1}(\Omega)$ для любого $\gamma>n /(n-p)$, а согласно следствию 4.5 из [9] если $u$ - энтропийное решение задачи (4.1), то $|u|^{q}[\ln (2+|u|)]^{-n /(n-p)}[\ln \ln (3+|u|)]^{-\gamma} \in L^{1}(\Omega)$ для любого $\gamma>n /(n-p)$. Как видно, предложения 3 и 4 являются более сильными результатами по сравнению с упомянутыми результатами работ [8;9] в применении к решениям задачи (4.1).

Предложение 5. Пусть $\lambda>0$ u $f[\ln (1+|f|)]^{\lambda} \in L^{1}(\Omega)$. Пусть и - энтропийное решение задачи (4.1). Тогда

$$
\forall k \geqslant e \quad \operatorname{meas}\{|u|>k\} \leqslant C k^{-q}(\ln k)^{-\lambda n /(n-p)},
$$

где $C$ - положительная константа, зависящая только от $n, p$, meas $\Omega, c_{2}, \lambda$ и нормы функизи $f[\ln (1+|f|)]^{\lambda}$ в $L^{1}(\Omega)$.

Д о к а з а т е л ь с т в о. Положим

$$
\gamma=\frac{p-1}{2 p}, \quad c_{0}=\frac{1}{\gamma^{\lambda}} \int_{\Omega}|f|[\ln (1+|f|)]^{\lambda} d x .
$$

Из предложения 2 вытекает, что

$$
\forall k>0 \quad \operatorname{meas}\{|u|>k\} \leqslant c k^{-q}\left(k^{-1 / 2}+\int_{\left\{|f| \geqslant k^{\gamma}\right\}}|f| d x\right)^{n /(n-p)} .
$$

Теперь положим $C=c\left[(2 \lambda)^{\lambda}+c_{0}\right]^{n /(n-p)}$ и зафиксируем произвольное $k \geqslant e$. Для любого $x \in\left\{|f| \geqslant k^{\gamma}\right\}$ имеем $|f(x)| \leqslant(\gamma \ln k)^{-\lambda}|f(x)|[\ln (1+|f(x)|)]^{\lambda}$. Следовательно,

$$
\int_{\left\{|f| \geqslant k^{\gamma}\right\}}|f| d x \leqslant c_{0}(\ln k)^{-\lambda} .
$$

Отсюда и из (4.3), учитывая неравенство $(\ln k)^{\lambda}<(2 \lambda)^{\lambda} k^{1 / 2}$, выводим, что meas $\{|u|>k\} \leqslant$ $C k^{-q}(\ln k)^{-\lambda n /(n-p)}$.

Используя следствие 1 и предложение 5, получаем такие результаты. 
Предложение 6. Пусть $\lambda \in(0,(n-p) / n]$ u $f[\ln (1+|f|)]^{\lambda} \in L^{1}(\Omega)$. Пусть $u$ - энтропийное решение задачи (4.1). Тогда для любого $\gamma>1-\lambda n /(n-p)$ имеем $|u|^{q}[\ln (2+|u|)]^{-\gamma} \in L^{1}(\Omega)$.

Предложение 7. Пусть $p>2-1 / n$. Пусть $\lambda \in(0,(n-p) / n]$ u $f[\ln (1+|f|)]^{\lambda} \in L^{1}(\Omega)$. Тогда существует слабое решение и задачи (4.1) такое, что для любого $\gamma>1-\lambda n /(n-p)$ имеем $|u|^{q}[\ln (2+|u|)]^{-\gamma} \in L^{1}(\Omega)$.

Используя следствие 2 и предложение 5 , приходим к следующим результатам.

Предложение 8. Пусть $\lambda>(n-p) / n u f[\ln (1+|f|)]^{\lambda} \in L^{1}(\Omega)$. Пусть $u-$ энтропийное решение задачи (4.1). Тогда для любого $\gamma \in(0, \lambda n /(n-p)-1)$ имеем $|u|^{q}[\ln (1+|u|)]^{\gamma} \in L^{1}(\Omega)$.

Предложение 9. Пусть $p>2-1 / n$. Пусть $\lambda>(n-p) / n$ u $f[\ln (1+|f|)]^{\lambda} \in L^{1}(\Omega)$. Тогда существует слабое решение и задачи (4.1) такое, что для любого $\gamma \in(0, \lambda n /(n-p)-1)$ имеем $|u|^{q}[\ln (1+|u|)]^{\gamma} \in L^{1}(\Omega)$.

В дополнение к этим предложениям имеем следующий результат.

Предложение 10. Пусть $p=2-1 / n$. Пусть $\lambda>(n-1) / n$ u $f[\ln (1+|f|)]^{\lambda} \in L^{1}(\Omega)$. Тогда существует слабое решение и задачи (4.1) такое, что для любого $\gamma \in\left(0, \lambda n^{2} /(n-1)^{2}-1\right)$ имеем $|u|^{n /(n-1)}[\ln (1+|u|)]^{\gamma} \in L^{1}(\Omega)$.

Д о к а з а т е л ь с т в о. Пусть $\tilde{f}:[0,+\infty) \rightarrow \mathbb{R}-$ функция такая, что для любого $s \in$ $[0,+\infty)$

$$
\tilde{f}(s)=\int_{\{|f| \geqslant s\}}|f| d x .
$$

Для любого $s>1$ имеем $\tilde{f}(s) \leqslant(\ln s)^{-\lambda}\left\|f[\ln (1+|f|)]^{\lambda}\right\|_{L^{1}(\Omega)}$. Отсюда ввиду того что $\lambda>$ $(n-1) / n$, вытекает неравенство

$$
\int_{1}^{+\infty} \frac{1}{s}[\tilde{f}(s)]^{n /(n-1)} d s<+\infty .
$$

Тогда в силу теоремы 3.2 из [4] для энтропийного решения $и$ задачи (4.1) справедливо включение $|\delta u| \in L^{n(p-1) /(n-1)}(\Omega)$. Следовательно, учитывая равенство $p=2-1 / n$, имеем $|\delta u| \in L^{1}(\Omega)$. Поэтому (см. [4, с. 1888]) и есть слабое решение задачи (4.1). Из предложения 8 и равенств $p=2-1 / n$ и $q=n(p-1) /(n-p)$ выводим, что для любого $\gamma \in\left(0, \lambda n^{2} /(n-1)^{2}-1\right)$ справедливо включение $|u|^{n /(n-1)}[\ln (1+|u|)]^{\gamma} \in L^{1}(\Omega)$.

Теперь рассмотрим случай более слабой интегрируемости функции $f$.

Предложение 11. Пусть $\lambda>0 u f[\ln \ln (e+|f|)]^{\lambda} \in L^{1}(\Omega)$. Пусть $u-$ энтропийное решение задачи (4.1). Тогда

$$
\forall k \geqslant 3 \quad \operatorname{meas}\{|u|>k\} \leqslant C k^{-q}(\ln \ln k)^{-\lambda n /(n-p)},
$$

где $C$ - положительнал константа, зависящая только от $n, p$, meas $\Omega, c_{2}, \lambda$ и нормы функи, ии $f[\ln \ln (e+|f|)]^{\lambda}$ в $L^{1}(\Omega)$.

Д о к а з а т ел ь с т в о. Положим

$$
\gamma=\frac{p-1}{2 p}, \quad c_{0}=2^{\lambda} \int_{\Omega}|f|[\ln \ln (e+|f|)]^{\lambda} d x
$$


и зафиксируем произвольное $k \geqslant e^{1 / \gamma^{2}}$. Учитывая неравенство $2 \ln (1 / \gamma) \leqslant \ln \ln k$, для любого $x \in\left\{|f| \geqslant k^{\gamma}\right\}$ имеем $|f(x)| \leqslant(\ln \ln k)^{-\lambda} 2^{\lambda}|f(x)|[\ln \ln (e+|f(x)|)]^{\lambda}$. Следовательно,

$$
\int_{\left\{|f| \geqslant k^{\gamma}\right\}}|f| d x \leqslant c_{0}(\ln \ln k)^{-\lambda} .
$$

Отсюда и из предложения 2 выводим, что

$$
\operatorname{meas}\{|u|>k\} \leqslant c\left[(2 \lambda)^{\lambda}+c_{0}\right]^{n /(n-p)} k^{-q}(\ln \ln k)^{-\lambda n /(n-p)} .
$$

Таким образом, если $k \geqslant e^{1 / \gamma^{2}}$, то справедливо неравенство (4.4). Если же $3 \leqslant k<e^{1 / \gamma^{2}}$, то

$$
\begin{aligned}
\operatorname{meas}\{|u|>k\} & \leqslant \operatorname{meas} \Omega=(\operatorname{meas} \Omega) k^{q}(\ln \ln k)^{\lambda n /(n-p)} k^{-q}(\ln \ln k)^{-\lambda n /(n-p)} \\
& \leqslant(\operatorname{meas} \Omega) e^{(q+\lambda n /(n-p)) / \gamma^{2}} k^{-q}(\ln \ln k)^{-\lambda n /(n-p)}
\end{aligned}
$$

В итоге приходим к требуемому заключению.

Используя следствие 3 и предложение 11, получаем такие результаты.

Предложение 12. Пусть $\lambda \in(0,(n-p) / n]$ u $f[\ln \ln (e+|f|)]^{\lambda} \in L^{1}(\Omega)$. Пусть $u-э н-$ тропийное решение задачи (4.1). Тогда для любого $\gamma>1-\lambda n /(n-p)$ справедливо включение $|u|^{q}[\ln (2+|u|)]^{-1}[\ln \ln (3+|u|)]^{-\gamma} \in L^{1}(\Omega)$.

Предложение 13. Пусть $p>2-1 / n$. Пусть $\lambda \in(0,(n-p) / n]$ u $f[\ln \ln (e+|f|)]^{\lambda} \in L^{1}(\Omega)$. Тогда существует слабое решение и задачи (4.1) такое, что для любого $\gamma>1-\lambda n /(n-p)$ имеем $|u|^{q}[\ln (2+|u|)]^{-1}[\ln \ln (3+|u|)]^{-\gamma} \in L^{1}(\Omega)$.

Используя следствие 4 и предложение 11, получаем такие результаты.

Предложение 14. Пусть $\lambda>(n-p) / n$ u $f[\ln \ln (e+|f|)]^{\lambda} \in L^{1}(\Omega)$. Пусть и - энтропийное решение задачи (4.1). Тогда для любого $\gamma \in(0, \lambda n /(n-p)-1)$ справедливо включение $|u|^{q}[\ln (2+|u|)]^{-1}[\ln \ln (3+|u|)]^{\gamma} \in L^{1}(\Omega)$.

Предложение 15. Пусть $p>2-1 / n$. Пусть $\lambda>(n-p) / n$ u $f[\ln \ln (e+|f|)]^{\lambda} \in L^{1}(\Omega)$. Тогда существует слабое решение и задачи (4.1) такое, что для любого $\gamma \in(0, \lambda n /(n-p)-1)$ имеем $|u|^{q}[\ln (2+|u|)]^{-1}[\ln \ln (3+|u|)]^{\gamma} \in L^{1}(\Omega)$.

\section{СПИСОК ЛИТЕРАТУРЫ}

1. Talenti G. Elliptic equations and rearrangements // Ann. Sc. Norm. Super. Pisa. Cl. Sci. (4). 1976. Vol. 3, no. 4. P. $697-718$.

2. Bénilan Ph., Boccardo L., Gallouët T., Gariepy R., Pierre M., Vazquez J.L. An $L^{1}$-theory of existence and uniqueness of solutions of nonlinear elliptic equations // Ann. Sc. Norm. Super. Pisa. Cl. Sci. (4). 1995. Vol. 22, no. 2. P. 241-273.

3. Ковалевский А.А. О суммируемости решений нелинейных эллиптических уравнений с правыми частями из классов, близких к $L^{1} / /$ Мат. заметки. 2001. Т. 70, № 3. С. 375-385.

4. Kovalevsky A.A. General conditions for limit summability of solutions of nonlinear elliptic equations with $L^{1}$-data // Nonlinear Anal. 2006. Vol. 64, no. 8. P. 1885-1895. doi: 10.1016/j.na.2005.08.008.

5. Boccardo L., Gallouet T. Nonlinear elliptic equations with right hand side measures // Commun. Partial Diff. Eq. 1992. Vol. 17, no. 3-4. P. 641-655. doi: 10.1080/03605309208820857.

6. Ковалевский А.А., Скрыпник И.И., Шишков А.Е. Сингулярные решения нелинейных эллиптических и параболических уравнений. Киев: Наукова думка, 2010. 499 с.

7. Boccardo L., Gallouet T. $W_{0}^{1,1}$ solutions in some borderline cases of Calderon-Zygmund theory // J. Diff. Eq. 2012. Vol. 253, no. 9. P. 2698-2714. doi: 10.1016/j.jde.2012.07.003. 
8. Boccardo L., Gallouet T. Summability of the solutions of nonlinear elliptic equations with right hand side measures // J. Convex Anal. 1996. Vol. 3, no. 2. P. 361-365.

9. Ковалевский А.А. Априорные свойства решений нелинейных уравнений с вырождающейся коэрцитивностью и $L^{1}$-данными // Современная математика. Фундаментальные направления. 2006. T. 16. C. $47-67$.

Ковалевский Александр Альбертович

Поступила 16.10.2018

После доработки 1.11.2018

Принята к публикации 5.11.2018

д-р физ.-мат. наук, профессор,

ведущий науч. сотрудник

Институт математики и механики им. Н.Н. Красовского УрО РАН;

Уральский федеральный университет

г. Екатеринбург

e-mail: alexkv171@mail.ru

\section{REFERENCES}

1. Talenti G. Elliptic equations and rearrangements. Ann. Sc. Norm. Super. Pisa. Cl. Sci. (4), 1976, vol. 3, no. 4 , pp. $697-718$.

2. Bénilan Ph., Boccardo L., Gallouët T., Gariepy R., Pierre M., Vazquez J.L. An $L^{1}$-theory of existence and uniqueness of solutions of nonlinear elliptic equations. Ann. Sc. Norm. Super. Pisa. Cl. Sci. (4), 1995, vol. 22, no. 2, pp. 241-273.

3. Kovalevskii A.A. Integrability of solutions of nonlinear elliptic equations with right-hand sides from classes close to $L^{1}$. Math. Notes, 2001, vol. 70, no. 3, pp. 337-346. doi: 10.1023/A:1012343728148.

4. Kovalevsky A.A. General conditions for limit summability of solutions of nonlinear elliptic equations with $L^{1}$-data. Nonlinear Anal., 2006, vol. 64, no. 8, pp. 1885-1895. doi: 10.1016/j.na.2005.08.008.

5. Boccardo L., Gallouet T. Nonlinear elliptic equations with right hand side measures. Commun. Partial Diff. Eq., 1992, vol. 17, no. 3-4, pp. 641-655. doi: 10.1080/03605309208820857.

6. Kovalevsky A.A., Skrypnik I.I., Shishkov A.E. Singular solutions of nonlinear elliptic and parabolic equations. Berlin: De Gruyter, 2016, De Gruyter Ser. Nonlinear Anal. Appl., vol. 24, 434 p. Original Russian text published in Singulyarnye resheniya nelineinykh ellipticheskikh i parabolicheskikh uravnenii, Kiev: Naukova Dumka, 2010, 499 p. doi: 10.1515/9783110332247.

7. Boccardo L., Gallouet T. $W_{0}^{1,1}$ solutions in some borderline cases of Calderon-Zygmund theory. J. Diff. Eq., 2012, vol. 253, no. 9, pp. 2698-2714. doi: 10.1016/j.jde.2012.07.003 .

8. Boccardo L., Gallouet T. Summability of the solutions of nonlinear elliptic equations with right hand side measures. J. Convex Anal., 1996, vol. 3, no. 2, pp. 361-365.

9. Kovalevsky A.A. A priori properties of solutions of nonlinear equations with degenerate coercivity and $L^{1}$-data. J. Math. Sci., 2008, vol. 149, no. 5, pp. 1517-1538. doi: 10.1007/s10958-008-0080-6 .

Received October 16, 2018

Revised November 1, 2018

Accepted November 5, 2018

Funding Agency: This work was supported by the Russian Academic Excellence Project (agreement no. 02.A03.21.0006 of August 27, 2013, between the Ministry of Education and Science of the Russian Federation and Ural Federal University).

Aleksandr Al'bertovich Kovalevsky, Dr. Phys.-Math. Sci., Prof., Krasovskii Institute of Mathematics and Mechanics of the Ural Branch of the Russian Academy of Sciences, Yekaterinburg, 620108 Russia; Institute of Natural Sciences and Mathematics, Ural Federal University, Yekaterinburg, 620002 Russia, e-mail: alexkvl71@mail.ru. 